\title{
Cumulative Balance Testing of Logic Circuits
}

\author{
Krishnendu Chakrabarty, Student Member, IEEE, and John P. Hayes, Fellow, IEEE
}

\begin{abstract}
We present a new test response compression method called cumulative balance testing (CBT) that extends both balance testing and accumulator compression testing. CBT uses an accumulated balance signature, and it guarantees very high error coverage (over 99\%) for various error models. We demonstrate that the single stuck-line (SSL) fault coverage of CBT for many of the ISCAS 85 combinational benchmark circuits is $100 \%$, and for al] but one circuit, the fault coverage is over $99.5 \%$. To make processor circuits self-testing, any existing accumulators and counters can be exploited to implement CBT. Its ease of implementation, provably high error coverage, and exceptionally high SSL fault coverage, even with reduced (nonexhaustive) test sets, make CBT suitable for the built-in self testing of processor circuits that require a guaranteed level of test confidence.
\end{abstract}

\section{INTRODUCTION}

$\mathbf{M}$ ANY built-in self-testing (BIST) techniques compress the test response information into a compact form called a signature. Well-known examples are ones counting, transition counting, signature analysis, and syndrome testing [2]. The fundamental problem with these methods is aliasing, which occurs when a faulty response produces the fault-free signature, thereby causing a loss of fault coverage. By reducing the fault coverage, aliasing has an adverse impact on test confidence, especially when high fault coverage is required. In practice, SSL fault coverage is often mandated at a level of $99 \%$ or higher.

We propose a new approach to BIST called cumulative balance testing (CBT). It employs a compression scheme that extends both balance testing [5], [6] and accumulator compression testing [19], [3], and offers several advantages. The error coverage is exceptionally high (over 99\%) for different error models, even for short test lengths. The SSL fault coverage for all but four of the ISCAS 85 combinational benchmark circuits [4] is $100 \%$ This, combined with the ease of implementation and a short fault signature makes CBT very suitable for testing circuits that require a very high level of fault coverage.

We begin by briefly reviewing balance testing, a BIST implementation of which is shown in Fig. 1. A balanced function is true for exactly half its input combinations and a circuit realizing a balanced function is called a balanced circuit. As defined in [5], balance testing involves the application of exhaustive input patterns to a balanced circuit, and the use of an up-down counter for compressing the test response. The

Manuscript received September 16, 1993; revised September 6, 1994. This work was supported by the National Science Foundation under Grant MIP9200526.

The authors are with the Advanced Computer Architecture Laboratory, Department of Electrical Engineering and Computer Science, University of Michigan, Ann Arbor, MT 48109 USA.

IEEE Log Number 9408372.

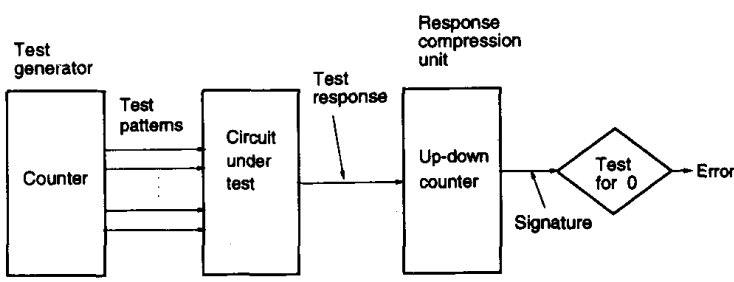

Fig. 1. A BIST implementation of balance testing.

counter is incremented whenever the test response is 1 , and decremented whenever the test response is 0 . For the fault-free circuit, the counter stores the value zero. A fault is detected if it makes the circuit unbalanced so that the up-down counter registers a nonzero value. The content of the up-down counter (zero in the fault-free case) is the balance signature of the test response.

Balance testing is not limited to balanced circuits only [6], nor does it require the use of all possible input patterns (exhaustive testing). To apply it to an unbalanced circuit, we can replace the up-down counter of Fig. 1 by a down counter preset to $|f|^{1}$, where $|f|^{1}$ is the number of minterms of the function $f$ realized by the circuit. The counter is decremented whenever the test response is 1 . Another aspect of balance testing not previously considered is that it can be used with pseudorandom and "reduced" test sets; exhaustive test sets and minterm computation are not. necessary to obtain good fault coverage, as we demonstrate in this paper. While pseudorandom patterns can be generated using linear feedback shift-registers [2], reduced test sets can be obtained using an ATPG program and applied using either a ROM and counter or a nonlinear feedback shift-register [23]. For nonexhaustive testing, the down counter of Fig. 1 is preset to the number of $1 \mathrm{~s}$ in the fault-free test response and, as before, it is decremented whenever the test response is 1 .

CBT uses the cumulative balance signature, obtained by accumulating balance signatures, and provides greater fault coverage than balance testing. In this paper, we discuss CBT for single-output circuits only. For circuits with multiple outputs, the CBT testing process can either be simply repeated for every observable output, or the outputs can be combined using a suitable space compaction function as in [7], [24] that guarantees error propagation.

The organization of this paper is as follows. In Section II, we introduce $\mathrm{CBT}$ and define the cumulative balance signature. Section III analyzes error detection in CBT. In Section IV, we calculate the error coverage for various error models. Finally in Section V, we present the experimental data on fault coverage of CBT for some representative logic circuits, including the ISCAS 85 benchmarks [4]. 


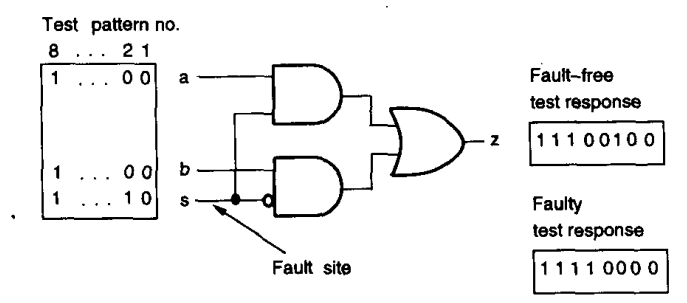

(a)

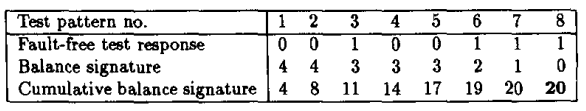

(b)

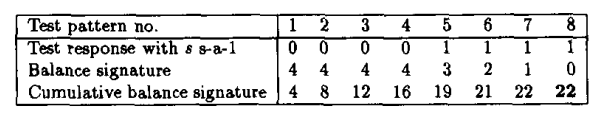

(c)

Fig. 2. CBT of a 2-to-1 multiplexer circuit. (a) Exhaustive test patterns applied to the logic circuit. (b) Fault-free signatures. (c) Signatures for the fault $s$ s-a- 1 .

\section{Cumulative Balance Signature}

We now introduce the notion of cumulative balance signature, which constitutes the compressed test response in CBT. The syndrome $K$ of a circuit for a given test set $T$ is the number of ones that appear in the output stream on application of all the test patterns in $T$. The accumulator syndrome, introduced by Saxena and Robinson for ACT [19], is obtained by accumulating syndromes over successive time periods from a test set containing $m$ patterns. The cumulative balance signature proposed here is obtained by accumulating balance signatures. Let $S_{i}$ be the balance signature after the application of $i$ test patterns. Similarly, let $C_{i}$ be the cumulative balance signature after the $i$ test patterns have been applied, that is, $C_{i}=C_{i-1}+S_{i}\left(\bmod m^{2}\right)$. In other words, $C_{i}=\sum_{j=1}^{i} S_{j}\left(\bmod m^{2}\right)$. The accumulator syndrome $A_{m}$ and the cumulative balance signature $C_{m}$ are related by the equation $A_{m}+C_{m}=m K$, where $K$ is the syndrome. This implies that a compression scheme which uses both the balance signature and the cumulative balance signature will provide exactly the same fault coverage as ACT. However, we show in this paper that CBT, which uses only the cumulative balance signature, provides almost the same fault coverage as ACT with a much shorter fault signature.

We first illustrate CBT with a simple example. Consider the 2-to-1 multiplexer circuit shown in Fig. 2(a) and tested exhaustively. With all $2^{3}$ test patterns applied in the order $a b s=000,001, \cdots, 111$, and the signature counter initialized to four, the fault-free test response, balance signature, and cumulative balance signature are as shown in Fig. 2(b). The faulty test response, balance signature, and cumulative balance signature for the fault $s$ s-a-1 are shown in Fig. 2(c). This fault is not detected by balance testing, but it is easily detected by CBT. Fig. 3 shows another circuit, taken from [5], where a balance-untestable fault is detected by CBT.

CBT uses an adder and a register, in addition to a counter, to compress the test response. When used to make processor
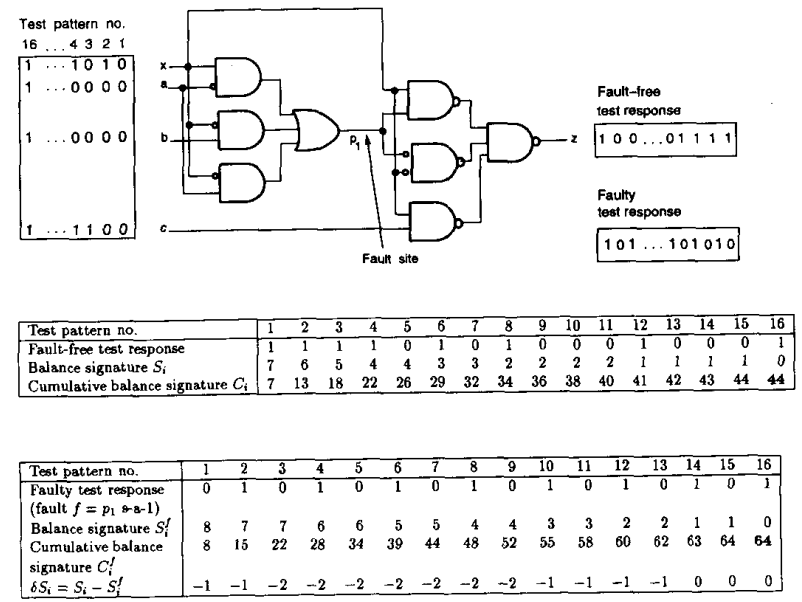

Fig. 3. A circuit with a balance-untestable fault $f=p_{1} \mathrm{~s}-\mathrm{a}-1$ that is detected by CBT.

circuits self-testing, any existing adders and accumulators can be exploited to implement CBT, as has been done for other accumulator-based BIST schemes [18]. For circuits that do not contain such common datapath modules, the hardware overhead associated with CBT may be high. Fig. 3 shows a representative BIST implementation of CBT. For $m$ test patterns, it suffices to use a $\left(2 \log _{2} m\right) \mathrm{b}$ adder, and therefore the additions are carried out modulo $m^{2}$. We show later that the modular addition does not reduce the fault coverage of CBT.

In $\mathrm{ACT}$, the fault signature consists of the syndrome of length $\log _{2} m$, and the accumulator syndrome of length $2 \log _{2} m$. A two-part signature is also used in group-theoretic signature analysis (GSTA) [3]. While the first part is once again the syndrome of length $\log _{2} m$, the second part is the accumulated syndrome modulo $m$, and is therefore of length $\log _{2} m$. For CBT, we propose the use of a single fault signature (the cumulative balance signature) which is of length $2 \log _{2} m$. In [19] and [3], error coverage is studied using partition theory and coding theory, respectively. In contrast, we analyze the error coverage in CBT using the central limit theorem of statistics, and apply the same method to compute the error coverage for ACT. We also present the actual CBT and ACT fault coverage for SSL faults in the ISCAS 85 benchmark circuits, which we obtained by simulation.

\section{ERROR DETECTION}

Error masking or aliasing occurs whenever an erroneous test response has the same signature as the error-free response. Two definitions of the aliasing probability have been widely used in the literature. The first [21] and more common one defines it as the probability that the test response is erroneous and has the same signature as the error-free test response. The second [8] defines aliasing probability as the conditional probability that the test response has the same signature as the error-free response, given that the test response is erroneous. As argued in [14], the first definition also includes the probability that the test generator detects the fault, i.e., it measures the efficiency 


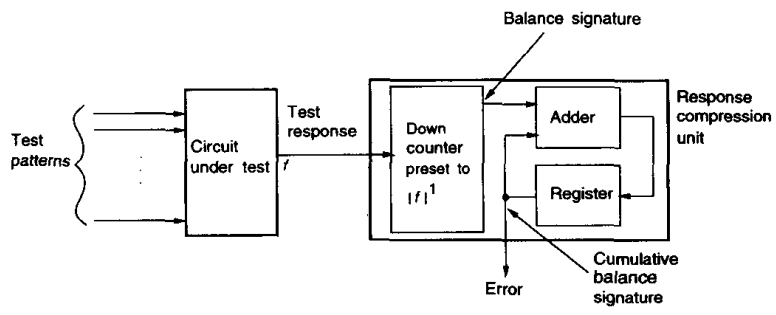

Fig. 4. The CBT scheme.

TABLE I

Difference Table Illustrating the Proof of Theorem 1

\begin{tabular}{|c|c|}
\hline \begin{tabular}{|l|} 
Test pattern \\
number $i$ \\
\end{tabular} & $\begin{array}{l}\text { Difference between fault-free } \\
\text { and faulty balance signatures, } \delta S_{i}\end{array}$ \\
\hline \begin{tabular}{|lllll}
1 & & & & \\
\end{tabular} & 0 \\
\hline 2 & 0 \\
\hline$i_{1}-1$ & \\
\hline $\begin{array}{l}i_{1} \\
i_{1}+1\end{array}$ & $\begin{array}{l}(-1)^{b_{1_{1}}} \\
(-1)^{b_{\mathrm{n}_{1}}}\end{array}$ \\
\hline$i_{2}-1$ & $(-1)^{b_{11}}$ \\
\hline $\begin{array}{l}i_{2} \\
i_{2}+1\end{array}$ & $\begin{array}{l}(-1)^{b_{1_{1}}}+(-1)^{b_{b_{2}}} \\
(-1)^{b_{b_{1}}}+(-1)^{b_{i_{2}}}\end{array}$ \\
\hline$i_{k}-1$ & $(-1)^{b_{11}}+(-1)^{b_{b_{2}}} \cdots(-1)^{b_{b_{k-1}}}$ \\
\hline $\begin{array}{l}i_{k} \\
i_{k}+1\end{array}$ & $\begin{array}{l}(-1)^{b_{1_{1}}}+(-1)^{b_{b_{2}}} \cdots(-1)^{b_{k_{k}}} \\
(-1)^{b_{1_{1}}}+(-1)^{b_{1_{2}}} \cdots(-1)^{b_{k_{k}}}\end{array}$ \\
\hline$\cdots$ & $(-1)^{b_{a_{1}}}+(-1)^{b_{i_{2}}} \ldots(-1)^{b_{k_{k}}}$ \\
\hline
\end{tabular}

of both test pattern generation and response compression. We adopt the second definition since we are concerned only with response compression. Furthermore, the use of complete test sets implies that the test generator's efficiency is $100 \%$, and guarantees that the test response of a faulty circuit is erroneous.

We now derive some useful error-detection properties of CBT.

Theorem 1: Let the fault-free response of the circuit under test be $b_{1} b_{2} \cdots b_{m}$. Let $k$ bits be in error in positions $i_{1}, i_{2}, \cdots, i_{k}$ respectively. The error is not detected by CBT if and only if $\sum_{j=1}^{k}(-1)^{b_{i_{j}}} i_{j}-(m+1) \sum_{j=1}^{k}(-1)^{b_{i_{j}}}=0$.

Proof: Let $\delta C_{m}=C_{m}-C_{m}^{f}$ be the difference between the fault-free and faulty cumulative balance signatures. Let $\delta S_{i}=S_{i}-S_{i}^{f}$ be the difference between the fault-free and faulty balance signatures for the first $i$ test patterns, as shown in Table I. Now, $\delta C_{m}=\sum_{j=1}^{m} \delta S_{i}$, hence adding the entries in the $\delta S_{i}$ column of the difference table, we get

$\delta C_{m}$

$$
=\sum_{j=1}^{k-1}\left(i_{j+1}-i_{j}\right) \sum_{l=1}^{j-1}(-1)^{b_{i_{l}}}+\left(m+1-i_{k}\right) \sum_{l=1}^{m}(-1)^{b_{i_{l}}} .
$$

This simplifies to

$$
\begin{aligned}
\delta C_{m} & =(-1)^{b_{i_{1}}} i_{1}+\cdots+(-1)^{b_{i_{k}}} i_{k}-(m+1) \sum_{j=1}^{k}(-1)^{b_{i_{j}}} \\
& =\sum_{j=1}^{k}(-1)^{b_{i_{j}}} i_{j}-(m+1) \sum_{j=1}^{k}(-1)^{b_{i_{j}}} .
\end{aligned}
$$

The error is not detected if and only if $\delta C_{m}=0$, i.e., $\sum_{j=1}^{k}(-1)^{b_{i}} i_{j}-(m+1) \sum_{j=1}^{k}(-1)^{b_{i_{j}}}=0$.
To illustrate Theorem 1, we return to the example circuit of Fig. 3. The errors are in bit positions 1, 3, 10, and 14, and $-1-3+10+14-0=20$, so the theorem implies that the fault is detected, which the figure confirms. As an illustration of a fault that is not detected by CBT, consider the 3-input exclusive-or gate with input lines $x_{1} x_{2} x_{3}$, and test patterns applied in the normal increasing order $x_{1} x_{2} x_{3}=000,001, \cdots$, 111. The fault-free response $b_{1} b_{2} \cdots b_{8}$ is 01101001 . Now suppose the input line $x_{3}$ is s-a- 0 , giving the faulty response stream 00111100 . The fault produces a $4 \mathrm{~b}$ error at the output, the error positions being $i_{1}=2, i_{2}=4, i_{3}=6$ and $i_{4}=8$. Since $\sum_{j=1}^{3}(-1)^{b_{i_{j}}} i_{j}-i_{4} \sum_{j=1}^{4}(-1)^{b_{i_{j}}}-\sum_{j=1}^{4}(-1)^{b_{i_{j}}}=$ $8-8+0=0$, the error, and therefore the corresponding fault, are not detected.

In ACT, an error is undetected only if the balance signature is zero, i.e., $\sum_{j=1}^{k}(-1)^{b_{i_{j}}}=0$. Therefore, setting $\sum_{j=1}^{k}(-1)^{b_{i_{j}}}=0$ in Theorem 1 , we see that error masking occurs in ACT if and only if $\sum_{j=1}^{k}(-1)^{b_{i_{j}}} i_{j}=0$.

Theorem 1 is the key result that we use later to calculate the error coverage. We also use the theorem to establish other useful error-detection properties of the cumulative balance signature. For example, Theorem 1 implies that the modulo- $m^{2}$ addition, i.e., using a $\left(2 \log _{2} m\right)$-b adder, does not introduce aliasing. Suppose $\delta C_{m}=0\left(\bmod m^{2}\right)$, i.e., $\delta C_{m}=p m^{2}$, where $p$ is an integer. From Lemma 2 in the appendix, we see that the only value of $p$ that causes aliasing is zero. This is in contrast to GSTA [3], where the use of modulo- $m$ addition reduces the fault coverage.

We next show that it suffices to use an adder with $2 \log _{2} m-$ $1 \mathrm{~b}$, and that this is a strict lower bound on the size of the adder. With such an adder, aliasing occurs if and only if $\delta C_{m}=0\left(\bmod m^{2} / 2\right)$, i.e. $\delta C_{m}=p m^{2} / 2$. This implies that $p$ is zero for all $m>1$. Next, suppose we use an adder with $2 \log _{2} m-2 \mathrm{~b}$. In this case, $\delta C_{m}$ can be equal to $m^{2} / 4$, therefore aliasing can occur for $p=1$.

In some count-based compression schemes like ones counting, syndrome counting, balance testing, and parity checking, the test patterns can be permuted without affecting the signature or aliasing characteristics. In others like transition counting, aliasing is sensitive to the test application order. In both CBT and ACT, error detection also depends on the test ordering.

Suppose $m$ test patterns are applied to the circuit under test in the order $T_{1} T_{2} \cdots T_{m}$. We next investigate the effect of changing the test application sequence to $T_{2} T_{3} \cdots T_{m} T_{1}$. The relative order of the test patterns remains unchanged, but the test application is now started with $T_{2}$. The following theorem shows that the ACT-detectable faults that are masked by CBT can be detected by a simple rotation of the test application sequence.

Theorem 2: If an error with nonzero balance signature is undetected by CBT for the test sequence $T_{1} T_{2} \cdots T_{m}$, then it is detected by CBT for the test sequence $T_{2} T_{3} \cdots T_{m} T_{1}$.

Proof: For the test sequence $T_{1} T_{2} \cdots T_{m}$, let the response bits in error be $b_{i_{1}}, \cdots, b_{i_{k}}$. From Theorem 1, we know that $\sum_{j=1}^{k}(-1)^{b_{i_{j}}} i_{j}=(m+1) \sum_{j=1}^{k}(-1)^{b_{i_{j}}}$. Next consider the test sequence $T_{2} T_{3} \cdots T_{m} T_{1}$. The following 
TABLE II

Five-Bit Erroneous Sequences with Nonzero Balance Signatures that are Detected by a One-Bit Rotation of the Test Sequence

\begin{tabular}{||l|l|l|l|l|l|l||}
\hline $\begin{array}{l}\text { Response } \\
\text { nequence } \\
\text { number }\end{array}$ & $\begin{array}{l}\text { Test } \\
\text { sequence }\end{array}$ & $\begin{array}{l}\text { Error-free } \\
\text { response } \\
\text { sequence }\end{array}$ & $\begin{array}{l}\text { Erroneous } \\
\text { response } \\
\text { sequence }\end{array}$ & $\begin{array}{l}\text { Error-free } \\
\text { cumulative } \\
\text { balance signature }\end{array}$ & $\begin{array}{l}\text { Erroneous } \\
\text { cumulative } \\
\text { balance signature }\end{array}$ & $\begin{array}{l}\text { Detected } \\
\text { by CBT? }\end{array}$ \\
\hline 1 & $T_{1} T_{2} T_{3} T_{4} T_{5}$ & 10000 & 00110 & 0 & 0 & No \\
& $T_{2} T_{3} T_{4} T_{5} T_{1}$ & 00001 & 01100 & 4 & -2 & Yes \\
2 & $T_{1} T_{2} T_{3} T_{4} T_{5}$ & 11000 & 01110 & 1 & 1 & No \\
& $T_{2} T_{3} T_{4} T_{5} T_{1}$ & 10001 & 11100 & 4 & -2 & Yes \\
3 & $T_{1} T_{2} T_{3} T_{4} T_{5}$ & 00110 & 10000 & 5 & 5 & Yo \\
4 & $T_{2} T_{3} T_{4} T_{5} T_{1}$ & 01100 & 00001 & 3 & 9 & No \\
& $T_{1} T_{2} T_{3} T_{4} T_{5}$ & 01110 & 11000 & 6 & 6 & 9 \\
\hline
\end{tabular}

TABLE III

ILlustration of an Undetectable Error that is Detected for Every Rotation of the Test SEquence

\begin{tabular}{||l|l|l|l|l|l||}
\hline $\begin{array}{l}\text { Test } \\
\text { sequence }\end{array}$ & $\begin{array}{l}\text { Error-free } \\
\text { response } \\
\text { sequence }\end{array}$ & $\begin{array}{l}\text { Erroneous } \\
\text { response } \\
\text { sequence }\end{array}$ & $\begin{array}{l}\text { Error-free } \\
\text { cumulative } \\
\text { balance signature }\end{array}$ & $\begin{array}{l}\text { Erroneous } \\
\text { cumulative } \\
\text { balance signature }\end{array}$ & $\begin{array}{l}\text { Detected } \\
\text { by CBT? }\end{array}$ \\
\hline$T_{1} T_{2} T_{3} T_{4} T_{5}$ & 11000 & 01110 & 1 & 1 & No \\
$T_{2} T_{3} T_{4} T_{5} T_{1}$ & 10001 & 11100 & 4 & -2 & Yes \\
$T_{3} T_{4} T_{5} T_{1} T_{2}$ & 00011 & 11001 & 7 & 0 & Yes \\
$T_{4} T_{5} T_{1} T_{2} T_{3}$ & 00110 & 10011 & 5 & 2 & Yes \\
$T_{5} T_{1} T_{2} T_{3} T_{4}$ & 01100 & 00111 & 3 & 4 & Yes \\
\hline
\end{tabular}

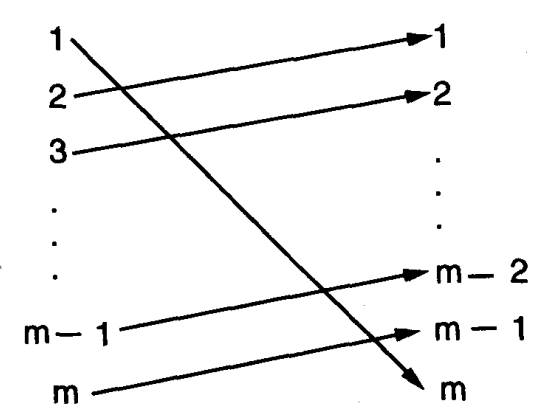

Fig. 5. Illustration of the mapping $i_{j} \rightarrow i_{j}-1(\bmod m)$ in the proof of Theorem 2.

mapping exists from error bit positions with test sequence $T_{1} T_{2} \cdots T_{m}$ to the error bit positions with test sequence $T_{2} T_{3} \cdots T_{m} T_{1}: i_{j} \rightarrow i_{j}-1(\bmod m), 1 \leq j \leq k$. This implies that if $i_{j} \geq 2, i_{j} \rightarrow i_{j}-1$, otherwise $i_{j} \rightarrow i_{j}-1+m$ (see Fig. 5). We need to consider the following two cases: Case 1: The first bit $i_{1}$ is not in error. In this case, the error remains undetected if $-\sum_{j=1}^{k}(-1)^{b_{i_{j}}}+\sum_{j=1}^{k}(-1)^{b_{i_{j}}} i_{j}=$ $\sum_{j=1}^{k}(-1)^{b_{i_{j}}} i_{j}$, a contradiction since $\sum_{j=1}^{k}(-1)^{b_{i_{j}}} \neq 0$. Case 2: The first bit $i_{1}$ is in error. In this case, the error is undetected if $-\sum_{j=1}^{k}(-1)^{b_{i_{j}}}+\sum_{j=1}^{k}(-1)^{b_{i_{j}}} i_{j}$ $+m(-1)^{b_{1}}=\sum_{j=1}^{k}(-1)^{b_{i_{j}}}$, i.e., $\sum_{j=1}^{k}(-1)^{b_{i_{j}}}=m(-1)^{b_{1}}$. Hence, $\sum_{j=1}^{k}(-1)^{b_{i_{j}}}$ is either $m$ (if $b_{1}=0$ ) or $-m$ (if $b_{1}=1$ ). This implies that the error-free response is all 1 's or all 0 's, and every response bit is in error, a contradiction.

For example, in Table II, we have enumerated all $5 \mathrm{~b}$ erroneous sequences with nonzero balance signatures that are not detected by CBT with the test sequence $T_{1} T_{2} T_{3} T_{4} T_{5}$. In each case, the error is detected by using the test sequence $T_{2} T_{3} T_{4} T_{5} T_{1}$. For rotations of more than one bit, most errors appear to be detected. For example, the erroneous sequence 01110 has a nonzero balance signature and the same cumulative balance signature as the corresponding error-free sequence 11000 . However, the error is detected for every rotation of the test sequence (Table III).

Although the fault coverage levels of CBT and ACT are almost equal (Sections IV and V), Theorem 2 shows that the fault coverage of CBT can be made exactly equal to that of $\mathrm{ACT}$ by changing the test application sequence. For exhaustive testing, this can be done by presetting the test generation counter to a different value. For ROM-based stored-pattern testing, we can preset the counter used to address the ROM. Finally, for LFSR-based pseudorandom test generation, we can use a different seed to achieve the same objective. Note that we are now effectively doubling the test application time and increasing the size of the reference signature to $4 \log _{2} m$. (The fault signature is still of size $2 \log _{2} m$.)

To see that not every permutation of the test sequence guarantees error detection, consider the test sequence $T_{1} T_{2} T_{3} T_{4} T_{5}$ in Table III where the error is not detected. For the test sequence $T_{1} T_{3} T_{5} T_{2} T_{4}$, the error-free and erroneous sequences are 10010 and 01011 , respectively. The cumulative balance signature for both these sequences is 3 , therefore the error remains undetected. We next consider undetectable errors that have a balance signature of zero. These errors are also undetected by ACT.

Theorem 3: If an error with zero balance signature is undetected by CBT for the test sequence $T_{1} T_{2} \cdots T_{m}$, then it remains undetected for the test sequence $T_{2} T_{3} \cdots T_{m} T_{1}$ if and only if the first response bit $i_{1}$ is not in error for the test sequence $T_{1} T_{2} \cdots T_{m}$. 
TABLE IV

Illustration of THEorem 3 for Two 6 b Sequences where (a) THE First Bit is not in Error and (b) the First Bit is in Error

\begin{tabular}{||l|l|l|l|l|l||}
\hline Test sequence & $\begin{array}{l}\text { Error-free } \\
\text { sequence }\end{array}$ & $\begin{array}{l}\text { Erroneous } \\
\text { sequence }\end{array}$ & $\begin{array}{l}\text { Error-free cumulative } \\
\text { balance signature }\end{array}$ & $\begin{array}{l}\text { Erroneous cumulative } \\
\text { balance signature }\end{array}$ & $\begin{array}{l}\text { Detected } \\
\text { by CBT? }\end{array}$ \\
\hline$T_{1} T_{2} T_{3} T_{4} T_{5} T_{6}$ & 101100 & 110010 & 5 & 5 & No \\
$T_{2} T_{3} T_{4} T_{5} T_{6} T_{1}$ & 011001 & 100101 & 8 & 8 & No \\
\hline
\end{tabular}

(a)

\begin{tabular}{||l|l|l|l|l|l||}
\hline Test sequence & $\begin{array}{l}\text { Error-free } \\
\text { sequence }\end{array}$ & $\begin{array}{l}\text { Erroneous } \\
\text { sequence }\end{array}$ & $\begin{array}{l}\text { Error-free cumulative } \\
\text { balance signature }\end{array}$ & $\begin{array}{l}\text { Erroneous cumulative } \\
\text { balance signature }\end{array}$ & $\begin{array}{l}\text { Detected } \\
\text { by CBT? }\end{array}$ \\
\hline$T_{1} T_{2} T_{3} T_{4} T_{5} T_{6}$ & 011010 & 100110 & 7 & 7 & No \\
$T_{2} T_{3} T_{4} T_{5} T_{6} T_{1}$ & 110100 & 001101 & 4 & 10 & Yes \\
\hline
\end{tabular}

(b)

Proof: Suppose the first bit $i_{1}$ is not in error. As in Case 1 of the proof of Theorem 2, the error remains undetected if and only if $\sum_{j=1}^{k}(-1)^{b_{i_{j}}}=0$, a condition satisfied by the theorem statement. Next, suppose $i_{1}$ is in error. From Case 2 of the proof of Theorem 2, we get $0=\sum_{j=1}^{k}(-1)^{b_{i_{j}}} \neq m(-1)^{b_{1}}$, hence the error is detected.

Table IV shows two erroneous sequences with zero balance signatures that are not detected by CBT. In Table IV(a), the $4 \mathrm{~b}$ error remains undetected for the test sequence $T_{2} T_{3} T_{4} T_{5} T_{6} T_{1}$ because the first response bit is not in error. On the other hand, the $4 \mathrm{~b}$ error in Table IV(b) is detected because the first response bit is incorrect.

For the remainder of this section, we consider counterbased exhaustive testing with the test patterns applied exhaustively in the normal increasing sequence $x_{1} x_{2} \cdots x_{n}=$ $00 \cdots 00,00 \cdots 01, \cdots, 11 \cdots 10,11 \cdots 11$. The advantages of exhaustive testing include ease of test generation, the fact that all detectable combinational faults are sensitized, and amenability to formal analysis [1]. It can be applied to circuits with a large number of primary inputs by using partitioning techniques [12], [17] and pseudoexhaustive test patterns [11]. For example, the 74630 parity generator circuit [20] has 6 primary outputs, each of which depends on only 10 primary inputs. Therefore, each output can be exhaustively tested with $2^{10}=1024$ test patterns.

First we introduce the notion of the weight of an input. With the input ordering $x_{n} x_{n-1} \cdots x_{i} \cdots x_{1}$ in counter-based testing, the input $x_{i}$ has weight $2^{i-1}$. For example, with the ordering $x_{3} x_{2} x_{1}$, the inputs $x_{3}, x_{2}$ and $x_{1}$ have weights 4,2 and 1 respectively.

Lemma 1: Let $x$ be a primary input of the circuit under test realizing the function $F(x, X)$. The vector $x X=0 \mathrm{~V}$ is a test for the fault $x$ s-a-1 if and only if the vector $x X=1 V$ is a test for the fault $x$ s-a- 0 .

Theorem 4: Let $x$ be a primary input of the circuit under test. If the fault $x \mathrm{~s}-\mathrm{a}-0$ ( $\mathrm{s}-\mathrm{a}-1)$ has a nonzero balance signature and is not detected by CBT with the tests applied exhaustively in the normal increasing sequence, then the fault $x \mathrm{~s}-\mathrm{a}-1(\mathrm{~s}-\mathrm{a}-0)$ is detected by CBT with the same test sequence.

Proof: Let $f(x, X)$ be the function realized by the circuit under test and let the fault $x$ s-a-1 be undetected by CBT. Suppose $x$ has weight $\alpha$ in the test application sequence, where $\alpha=2^{u}$ for some $u \geq 0$. It takes the following values during the exhaustive test application: $\underbrace{00 \cdots 0}_{\alpha} \underbrace{11 \cdots 1}_{\alpha} \underbrace{00 \cdots 0}_{\alpha} \cdots \underbrace{11 \cdots 1}_{\alpha}$. Let the fault $x$ s-a-1 be ${ }^{\alpha}$ undetected by ${ }^{\alpha}$ CBT, and let $b_{i_{1}}, b_{i_{2}}, \cdots, b_{i_{k}}$ be the erroneous response bits. If the bit position $i$ corresponds to an input combination $x X=0 V$, then the bit position $i+\alpha$ corresponds to the input combination $1 V$. If bit $i$ is in error then $f(1, V) \neq f(0, V)$, which implies that $b_{i} \neq b_{i+\alpha}$. Therefore, $\sum_{j=1}^{k}(-1)^{b_{i_{j}}} i_{j}=(m+1) \sum_{j=1}^{k}(-1)^{b_{i_{j}}}$ and for all $1 \leq j \leq k, b_{i_{j}} \neq b_{i_{j}+\alpha}$. Next consider the fault $x$ s-a- 0 . From Lemma 1 , the error is now in bit positions $i_{1}+\alpha, i_{2}+\alpha, \cdots, i_{k}+\alpha$, and

$$
\begin{aligned}
\sum_{j=1}^{k}(-1)^{b_{i_{j}+\alpha}}\left(i_{j}+\alpha\right) & \\
& =\sum_{j=1}^{k}(-1)^{b_{i_{j}+\alpha}} i_{j}+\alpha \sum_{j=1}^{k}(-1)^{b_{i_{j}+\alpha}} \\
& =-\sum_{j=1}^{k}(-1)^{b_{i_{j}}} i_{j}-\alpha \sum_{j=1}^{k}(-1)^{b_{i_{j}}} \\
& =-(m+1) \sum_{j=1}^{k}(-1)^{b_{i_{j}}}-\alpha \sum_{j=1}^{k}(-1)^{b_{i_{j}}}
\end{aligned}
$$

Since $\sum_{j=1}^{k}(-1)^{b_{i_{j}}} \neq 0$ and $\alpha \geq 1$, the fault $x$ s-a- 0 is detected by CBT.

Theorem 5: Let $x$ be a primary input of the circuit under test. If the fault $x \mathrm{~s}-\mathrm{a}-0(x \mathrm{~s}-\mathrm{a}-1)$ has a zero balance signature and is not detected by CBT with tests applied exhaustively in the normal increasing sequence, then the fault $x \mathrm{~s}-\mathrm{a}-1(x \mathrm{~s}-\mathrm{a}-0)$ is also not detected by CBT with the same test sequence.

Proof: The proof is similar to that of Theorem 4, the only difference being that here $\sum_{j=1}^{k}(-1)^{b_{i}} i_{j}=0$. This accounts for the fact that the fault $x \mathrm{~s}-\mathrm{a}-1(x \mathrm{~s}-\mathrm{a}-0)$ is not detected.

We next ask whether errors that are not detected by the cumulative balance signature can be detected by permuting the interconnections between the output lines of the test generator and the inputs of the circuit under test, thereby implicitly reordering the test patterns. Fig. 6 shows such a reconfigurable interconnection. We consider the effect of pairwise interchange of inputs $x_{a}$ and $x_{b}$ on faults that are not detected by CBT. Suppose $\alpha_{a}$ and $\alpha_{b}$ are the weights of $x_{a}$ and $x_{b}$ respectively with $\alpha_{a}>\alpha_{b}$. 


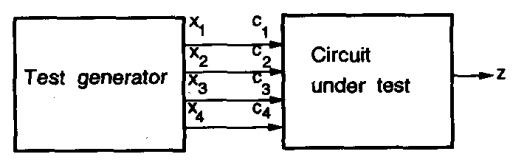

(a)

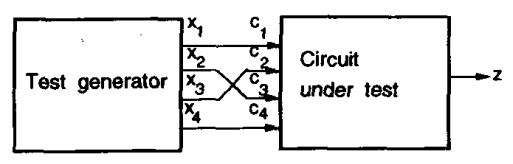

(b)

Fig. 6. Reconfigurable interconnections between the test generator and the circuit under test. (a) Input ordering $x_{1} x_{2} x_{3} x_{4}$. (b) Input ordering $x_{1} x_{3} x_{2} x_{4}$.

Theorem 6: Let $i_{1}, i_{2}, \cdots, i_{k}$ be the error positions in the response stream due to a fault that is not detected by CBT. Let $x_{a_{j}}$ be the value of the input variable $x_{a}$ in the input combination corresponding to position $i_{j}(1 \leq j \leq k)$. Let $x_{b_{j}}$ be defined similarly. The fault remains undetected by CBT after the interchange of inputs $x_{a}$ and $x_{b}$ if and only if

$$
\sum_{j=1}^{k}(-1)^{b_{i_{j}}}\left(1-\overline{x_{a_{j}} \oplus x_{b_{j}}}\right)(-1)^{x_{a_{j}}}=0 .
$$

Proof: Consider the error position $i_{j}$. If $x_{a_{j}}=x_{b_{j}}$ then the error position does not change after the transposition of inputs $x_{a}$ and $x_{b}$. Otherwise, the new error position is $i_{j}+\left(\alpha_{a}-\alpha_{b}\right)$ if $x_{a_{j}}=0$, and $i_{j}-\left(\alpha_{a}-\alpha_{b}\right)$ if $x_{a_{j}}=1$. Therefore, we can combine all these possibilities to get the following expression for the new error position $i_{j}^{\prime}$

$$
i_{j}^{\prime}=i_{j}+\left(\alpha_{a}-\alpha_{b}\right)\left(1-\overline{x_{a_{j}} \oplus x_{b_{j}}}\right)(-1)^{x_{a_{j}}} .
$$

Let $V=\sum_{j=1}^{k}(-1)^{b_{j}} i_{j}$. The fault remains undetected after the interchange if and only if

$$
\begin{aligned}
V & =\sum_{j=1}^{k}(-1)^{b_{i^{\prime}} i_{j}^{\prime}} \\
& =\sum_{j=1}^{k}(-1)^{b_{i_{j}}}\left\{i_{j}+\left(\alpha_{a}-\alpha_{b}\right)\left(1-\overline{x_{a_{j}} \oplus x_{b_{j}}}\right)(-1)^{x_{a_{j}}}\right\} .
\end{aligned}
$$

This implies that

$$
\left(\alpha_{a}-\alpha_{b}\right) \sum_{j=1}^{k}(-1)^{b_{i_{j}}}\left(1-\overline{x_{a_{j}} \oplus x_{b_{j}}}\right)(-1)^{x_{a_{j}}}=0
$$

i.e., $\sum_{j=1}^{k}(-1)^{b_{i j}}\left(1-\overline{x_{a_{j}} \oplus x_{b_{j}}}\right)(-1)^{x_{a_{j}}}=0$.

For example, in the 3-input exclusive-or gate with inputs $x_{1}, x_{2}, x_{3}$, the fault $x_{1}$ s-a- 0 is not detected with the input ordering $x_{1} x_{2} x_{3}$. The errors in the $8 \mathrm{~b}$ response are in bit positions $5,6,7,8$; therefore $i_{1}=5, i_{2}=6, i_{3}=7, i_{4}=8$. Suppose we switch inputs $x_{1}$ and $x_{3}$. Then $a=1, b=3$, and since $\sum_{i=5}^{8}(-1)^{b_{i}}\left(1-\overline{x_{1_{i}} \oplus x_{3_{i}}}\right)(-1)^{x_{1_{i}}}=0$, the fault $x_{1}$ s-a-0 remains undetected on interchanging $x_{1}$ and $x_{3}$.

Corollary 1: Let \# $\left(x_{a_{j}}=x_{b_{j}}, j=1 \cdots k\right)$ denote the number of times that $x_{a_{j}}=x_{b_{j}}$ for $1 \leq j \leq k$. If $\#\left(x_{a_{j}}=\right.$ $\left.x_{b_{j}}, j=1 \cdots k\right)$ is odd, a fault that is not detected by CBT becomes testable after the interchange of inputs $x_{a}$ and $x_{b}$.

Corollary 2: Let the undetected fault be a primary input $x_{a}$ s-a- $d, d \in\{0,1\}$. The fault remains undetected by
CBT on interchange of inputs $x_{a}$ and $x_{b}$ if and only if $\sum_{j=1}^{k}(-1)^{b_{i_{j}}}\left(1-x_{b_{j}}^{d}\right)=0$, where $x_{b_{j}}^{0}=x_{b_{j}}$ and $x_{b_{j}}^{1}=x_{b_{j}}^{\prime}$ Proof: We prove the corollary for $d=0$; the proof for $d=1$ is similar. Theorem 6 implies that the fault remains undetected if and only if $\sum_{j=1}^{k}(-1)^{b_{i_{j}}}\left(1-\overline{x_{a_{j}} \oplus \overline{x_{b_{j}}}}\right)(-1)^{x_{a_{j}}}=$ 0 . The output is in error only when $x_{a}=1$; therefore $x_{a_{j}}=1$, for $1 \leq j \leq k$, and the corollary follows.

\section{ERROR COVERAGE}

In this section, we calculate the asymptotic error coverage of ACT and CBT for the independent and asymmetric error models. We also investigate the coverage for dependent errors. For our analysis, we employ Theorem 1 and the central limit theorem of statistics [9]. This approach to calculating error coverage does not seem to have been used elsewhere.

An error model makes specific assumptions about the probability of occurrence of errors at the output of a faulty circuit that is exercised by test patterns. In our analysis, we employ the following error models:

1) The asymmetric error model [14], [22] assumes that the probability of an error depends on the fault-free circuit response. Given an SSL fault, a circuit's output value may change from 0 to 1 for some input patterns, from 1 to 0 for other patterns, and not change at all for others. The number of 1-to-0 changes generally does not equal the number of 0 to- 1 changes; and therefore the probability of the response bit being in error depends on the value of the fault-free function. Experimental results provided in [22] for the $74181 \mathrm{ALU}$ and the 432 ISCAS benchmark circuit support this model.

2) The independent error model [21] assumes nonequiprobable errors that are independent in time, i.e., the probability $\alpha$ that a response bit is in error is independent of the probability that other response bits are also in error. It is therefore a special case of the asymmetric error model. The independent error model is claimed to be particularly suitable for pseudorandom testing [13].

3) Many faults produce errors that occur within some restricted neighborhood. We analyze the error coverage for two such dependent errors. An $(s, k)$-burst error is one where all erroneous bits are within $s$ consecutive bit positions and at most $k \mathrm{~b}$ are in error. We make the simplifying assumption that all the $k \mathrm{~b}$ of an $(s, k)$-burst error are in error, and call it a $k$-burst error. Burst errors often arise when the faulty portion of a system is exercised extensively during testing [16]. A $k$ b repeated-use error is one where the $k$ erroneous bits are in positions $i+p n_{1}, i+p n_{2}, \cdots, i+p n_{k}$, where $p$ is a power of 2 (typically the word size of a computer system). Such errors occur during data transfer, as well as during arithmetic and logical operations [16]. 


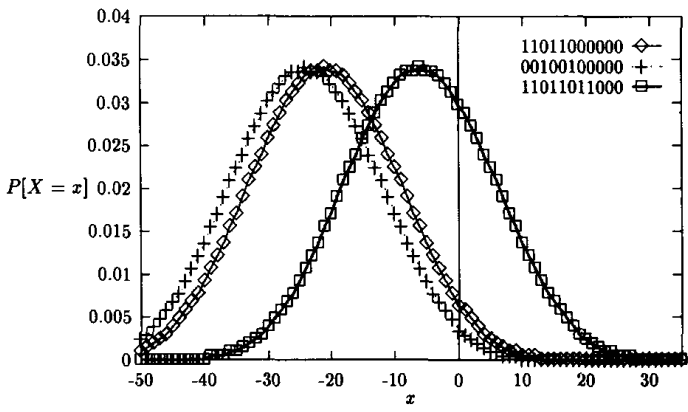

Fig. 7. The probabilities $P[X=x]$ obtained experimentally for three arbitrarily chosen fault-free sequences with $m=11$.

Let $\mathcal{E}_{1}$ be the event that the balance signature is zero, and let $\mathcal{E}_{2}$ be the event that the cumulative balance signature is identical to the reference signature. The error coverage of ACT is therefore $1-P\left[\mathcal{E}_{1} \cap \mathcal{E}_{2}\right]=1-P\left[\mathcal{E}_{1}\right] \mathcal{P}\left[\mathcal{E}_{2} \mid \mathcal{E}_{1}\right]$, where $P\left[\mathcal{E}_{2} \mid \mathcal{E}_{1}\right]=P\left[\sum_{j=1}^{k}(-1)^{b_{i_{j}}} i_{j}=0\right]$. Let $\mathcal{E}_{1}^{i}$ be the event that $\sum_{j=1}^{k}(-1)^{b_{i_{j}}}=i$, and let $\mathcal{E}_{3}$ be the event $\sum_{j=1}^{k}(-1)^{b_{i_{j}}} i_{j}=$ $(m+1) \sum_{j=1}^{k}(-1)^{b_{i_{j}}}$. Then the error coverage for CBT is $1-P\left[\mathcal{E}_{2}\right]=1-\sum_{i=-m}^{m} P\left[\mathcal{E}_{1}^{i}\right] P\left[\mathcal{E}_{3} \mid \mathcal{E}_{1}^{i}\right]$. We first describe a method to compute the conditional probability $P\left[\mathcal{E}_{2} \mid \mathcal{E}_{1}\right]$ and thereby the error coverage for ACT. The analysis will be later extended to CBT. For each response bit $b_{i}(1 \leq i \leq m)$, we define a random variable $X_{i}$ as follows

$$
X_{i}= \begin{cases}(-1)^{b_{i}} i, & \text { if bit } i \text { is in error } \\ 0, & \text { otherwise. }\end{cases}
$$

We are interested in the distribution of the sum $X=\sum_{i=1}^{m} X_{i}$ of these random variables, since $P\left[\mathcal{E}_{2} \mid \mathcal{E}_{1}\right]=P[X=0]$ from Theorem 1. For the independent and asymmetric error models, and a combinational circuit, the $X_{i}$ 's are independent, and therefore from the central limit theorem of statistics [9], $X$ follows a normal distribution. We show in the appendix that the central limit applies to our problem for both the asymmetric and independent error models.

To verify experimentally that $X$ can be approximated by the normal distribution, we computed the probabilities $P[X=x]$ assuming equiprobable errors for three different, arbitrarily chosen fault-free response sequences of lengths 9,10 , and 11 . In Fig. 7, we see that, for $m=11$, the normal distribution is a good approximation for the random variable $X$. The probability distributions for $m=9$ and $m=10$ are given in the appendix. We have also verified that, as expected, the approximation is even better for larger values $m$. We next use the normal distribution to derive the probability $P\left[\mathcal{E}_{2} \mid \mathcal{E}_{1}\right]$ for the asymmetric and independent error models. Let $X_{i}$ have mean $\mu_{i}$ and variance $\sigma_{i}^{2}$, and let $\mu$ and $\sigma$ be the corresponding measures for $X$.

1) Asymmetric Error Model: Let $\theta_{0}\left(\theta_{1}\right)$ be the probability that a response bit is in error given that its fault-free value is 0 (1). This implies that $P\left[X_{i}=i\right]=\theta_{1}, P\left[X_{i}=-i\right]=\theta_{0}$, and $P\left[X_{i}=0\right]=1-\theta_{1}-\theta_{0}$. Therefore, $\mu_{i}=i\left(\theta_{1}-\theta_{0}\right)$ and $\sigma_{i}^{2}=i^{2}\left(\theta_{1}+\theta_{0}+2 \theta_{1} \theta_{0}-\theta_{1}^{2}-\theta_{0}^{2}\right)$. This gives us the following equations for $\mu$ and $\sigma^{2}$

$$
\begin{aligned}
\mu & =\sum_{i=1}^{m} \mu_{i} \\
& =\frac{m(m+1)}{2}\left(\theta_{1}-\theta_{0}\right) \\
\sigma^{2} & =\sum_{i=1}^{m} \sigma_{i}^{2} \\
& =\frac{m(m+1)(2 m+1)}{6}\left(\theta_{1}+\theta_{0}+2 \theta_{1} \theta_{0}-\theta_{1}^{2}-\theta_{0}^{2}\right) .
\end{aligned}
$$

Since $X$ follows the normal distribution, its probability density function is given by

$$
f_{X}(x)=\frac{1}{\sqrt{2 \pi} \sigma} \exp \left(-\frac{(x-\mu)^{2}}{2 \sigma^{2}}\right)
$$

For a continuous random variable, the probability associated with an event $[X=c]$ is zero. However, the underlying random variable in this case is discrete, therefore we can approximate $P\left[\mathcal{E}_{2} \mid \mathcal{E}_{1}\right]=P[X=0]$ by the probability $P[-1 \leq$ $X \leq 1]$, i.e.

$$
P\left[\mathcal{E}_{2} \mid \mathcal{E}_{1}\right] \approx \int_{-1}^{1} \frac{1}{\sqrt{2 \pi} \sigma} \exp \left(-\frac{(x-\mu)^{2}}{2 \sigma^{2}}\right) d x .
$$

We next calculate $P\left[\mathcal{E}_{1}\right]$. Let $m=m_{0}+m_{1}$, where $m_{1}\left(m_{0}\right)$ is the number of response bits that are $1(0)$ in the fault-free case. Let $m^{\prime}=\min \left(m_{0}, m_{k}\right)$ and $k^{\prime}=k / 2$. Then

$$
\begin{aligned}
& P\left[\mathcal{E}_{1}\right] \\
& =\sum_{k^{\prime}=1}^{m^{\prime}} \theta_{1}^{k}\left(\begin{array}{c}
m_{1} \\
k^{\prime}
\end{array}\right) \theta_{0}^{k}\left(\begin{array}{c}
m_{0} \\
k^{\prime}
\end{array}\right)\left(1-\theta_{1}\right)^{m_{1}-k^{\prime}}\left(1-\theta_{0}\right)^{m_{0}-k^{\prime}} \\
& \leq \sum_{k^{\prime}=1}^{m / 2} \theta_{1}^{k}\left(\begin{array}{c}
m / 2 \\
k^{\prime}
\end{array}\right) \theta_{0}^{k}\left(\begin{array}{c}
m / 2 \\
k^{\prime}
\end{array}\right)\left(1-\theta_{1}\right)^{m / 2-k^{\prime}}\left(1-\theta_{0}\right)^{m / 2-k^{\prime}}
\end{aligned}
$$

since $P\left[\mathcal{E}_{1}\right]$ is maximum for $m_{1}=m_{0}=m^{\prime}=m / 2$.

2) Independent Error Model: This is a special case of the asymmetric error model with $\theta_{0}=\theta_{1}=\alpha$. From equations (2) and (3), we get $\mu=0$ and $\sigma^{2}=\frac{2 m(m+1)(2 m+1)}{3} \alpha^{2}$. Therefore

$$
P\left[\mathcal{E}_{2} \mid \mathcal{E}_{1}\right] \approx \int_{-1}^{1} \frac{1}{\sqrt{2 \pi} \sigma} \exp \left(-\frac{x^{2}}{2 \sigma^{2}}\right) d x
$$

and

$$
\begin{aligned}
P\left[\mathcal{E}_{1}\right] & =\sum_{k^{\prime}=1}^{m^{\prime}}\left(\begin{array}{c}
m_{1} \\
k^{\prime}
\end{array}\right)\left(\begin{array}{c}
m_{0} \\
k^{\prime}
\end{array}\right) \alpha^{2 k^{\prime}}(1-\alpha)^{m-2 k^{\prime}} \\
& \leq \sum_{k^{\prime}=1}^{m / 2}\left(\begin{array}{c}
m / 2 \\
k^{\prime}
\end{array}\right)\left(\begin{array}{c}
m / 2 \\
k^{\prime}
\end{array}\right) \alpha^{2 k^{\prime}}(1-\alpha)^{m-2 k^{\prime}} .
\end{aligned}
$$

The error coverage of ACT is given by $1-P\left[\mathcal{E}_{1} \cap \mathcal{E}_{2}\right]=1-$ $P\left[\mathcal{E}_{1}\right] P\left[\mathcal{E}_{2} \mid \mathcal{E}_{1}\right]$. Table $\mathrm{V}$ shows the error coverage for various values of $\theta_{0}, \theta_{1}$, and $\alpha$. In fact, the error coverage will generally be greater than these values because we overestimated 
TABLE V

Analytical Error Coverage (Percent) of ACT and CBT FOR THE ASYMMETRIC AND INDEPENDENT ERROR MODELS

\begin{tabular}{|c|c|c|c|c|c|c|c|c|}
\hline \multirow[b]{3}{*}{$\begin{array}{l}\text { Number of } \\
\text { tests } m\end{array}$} & \multicolumn{4}{|c|}{ ACT error coverage } & \multicolumn{4}{|c|}{ CBT error coverage } \\
\hline & \multicolumn{2}{|c|}{$\begin{array}{l}\text { Asymmetric error } \\
\text { model }\end{array}$} & \multicolumn{2}{|c|}{$\begin{array}{l}\text { Independent error } \\
\text { model }\end{array}$} & \multicolumn{2}{|c|}{$\begin{array}{l}\text { Asymmetric error } \\
\text { model }\end{array}$} & \multicolumn{2}{|c|}{$\begin{array}{l}\text { Independent error } \\
\text { model }\end{array}$} \\
\hline & & & $\alpha=0.2$ & $\alpha=0.4$ & $\begin{array}{l}\theta_{0}=0.1 \\
\theta_{1}=0.2\end{array}$ & $\begin{array}{l}\theta_{0}=0.4 \\
\theta_{1}=0.3\end{array}$ & $\alpha=0.2$ & $\alpha=0.4$ \\
\hline 16 & $\frac{1-0.2}{99.58}$ & $\frac{1-0 .}{99.79}$ & \begin{tabular}{|l|}
98.86 \\
\end{tabular} & \begin{tabular}{|l|}
59.48 \\
99
\end{tabular} & \begin{tabular}{|l|}
$1-0.2$ \\
97.73
\end{tabular} & \begin{tabular}{|l|l|}
$1-0.0$ \\
98.14
\end{tabular} & \begin{tabular}{|l|}
96.72 \\
\end{tabular} & \begin{tabular}{|l|}
97.75 \\
\end{tabular} \\
\hline 24 & 99.76 & 99.91 & 99.43 & 99.76 & 98.83 & 99.45 & 98.10 & \\
\hline 32 & $\begin{array}{l}99.84 \\
99.6\end{array}$ & 99.95 & 99.67 & 99.87 & $\begin{array}{l}89.00 \\
99.30\end{array}$ & $\begin{array}{l}99.68 \\
99.40\end{array}$ & 98.74 & 99.30 \\
\hline 40 & 99.90 & 99.97 & 99.79 & 99.91 & 99.58 & 99.80 & 98.74 & 99.21 \\
\hline 48 & 99.93 & 99.98 & 99.85 & 99.94 & 99.70 & 99.84 & 99.10 & 99.48 \\
\hline 56 & 0005 & 99.99 & 99.89 & 99.96 & 99.84 & 99. & 99.33 & 99.81 \\
\hline 100 & 99.98 & 99.99 & 99.97 & 99.99 & 99.89 & 99.99 & 99.48 & 99.97 \\
\hline
\end{tabular}

$P\left[\mathcal{E}_{1}\right]$ in equations (5) and (7) by taking $m_{1}=m_{0}=m / 2$. The analytical results of Table $\mathrm{V}$ demonstrate that ACT (and CBT, as we show below) guarantees extremely high error coverage. This confirms the results of [19] and is corroborated in Section V by the near-complete SSL fault coverage we obtained for the benchmark circuits.

We also studied the error coverage experimentally by exhaustively enumerating error patterns for $8 \leq m \leq 16$, and applying Theorem 1 to each pattern. Table VI lists the minimum error coverage for a fault-free sequence assuming equiprobable errors, and the average error coverage assuming that all fault-free sequences are equally likely. The experimental data closely matches the error coverage results obtained analytically.

We next calculate the error coverage for CBT. From Theorem 1 and the central limit theorem, the probability $P\left[\mathcal{E}_{3} \mid \mathcal{E}_{1}^{i}\right]=$ $P[X=i]$ is $\int_{i-1}^{i+1} f_{X}(x) d x$. Therefore, the error coverage is $\sum_{i=-m}^{m} P\left[\mathcal{E}_{1}^{i}\right] \int_{(m+1) i-1}^{(m+1) i+1} f_{X}(x) d x$. As in the analysis of ACT, the function $f_{X}(x)$ is obtained from the parameters of the error model. To calculate $P\left[\mathcal{E}_{1}^{i}\right]$, we let $m=m_{0}+m_{1}$, where $m_{1}\left(m_{0}\right)$ is the number of response bits that are $1(0)$ in the fault-free case. Let $m^{\prime}=\min \left(m_{0}, m_{k}\right)$ as before. Then, for the independent error model

$$
\begin{aligned}
P\left[\mathcal{E}_{1}^{i}\right] & =\sum_{j=0}^{m^{\prime}-i}\left(\begin{array}{c}
m_{0} \\
j
\end{array}\right)\left(\begin{array}{c}
m_{1} \\
j+i
\end{array}\right) \alpha^{j} \alpha^{j+i}(1-\alpha)^{m-2 j-i} \\
& \leq \sum_{j=0}^{m / 2-i}\left(\begin{array}{c}
m / 2 \\
j
\end{array}\right)\left(\begin{array}{c}
m / 2 \\
j+i
\end{array}\right) \alpha^{2 j+i}(1-\alpha)^{m-2 j-i}
\end{aligned}
$$

since $P\left[S_{m}=i\right]$ is maximum for $m_{1}=m_{0}=m^{\prime}=m / 2$. The asymmetric error model can be considered in similar fashion. The error coverage obtained in this way for CBT is listed in Table V. We see that the error coverage values for CBT and ACT are very close, indicating that the decrease in fault coverage, if any, due to dropping the balance signature is very small.

3) Dependent Error Models: We next analyze the error coverage for $k$-burst and repeated-use errors. We can no longer use the central limit theorem because the random variables $X_{1}, X_{2}, \cdots, X_{m}$ defined by (1) are not independent under these error models.

Theorem 7: Consider a $k$-burst error with zero balance signature and error bits $b_{\alpha+1}, b_{\alpha+2}, \cdots, b_{\alpha+k}$. Let $S$ be the set $\{1,2, \cdots, k\}$. The $k$-burst error is not detected by CBT
TABLE VI

ExPerimental ERror CoVerage (Percent) For the ASYMMETRIC AND INDEPENDENT ERROR MODELS

\begin{tabular}{||l|l|l|l|l||}
\hline \multirow{2}{*}{$\begin{array}{l}\text { Number of } \\
\text { tests } m\end{array}$} & \multicolumn{2}{|c|}{ ACT error coverage } & \multicolumn{2}{c|}{ CBT error coverage } \\
\cline { 2 - 5 } & Minimum & Average & Minimum & Average \\
\hline 8 & 98.05 & 99.11 & 96.48 & 98.76 \\
9 & 98.05 & 99.01 & 97.07 & 98.94 \\
10 & 98.24 & 99.13 & 97.36 & 99.08 \\
11 & 98.58 & 99.38 & 97.80 & 99.21 \\
12 & 98.68 & 99.97 & 98.07 & 99.31 \\
13 & 98.90 & 99.99 & 98.28 & 99.38 \\
14 & 98.98 & 99.99 & 98.49 & 99.46 \\
15 & 99.13 & 99.99 & 98.65 & 99.52 \\
16 & 99.21 & 99.99 & 98.79 & 99.59 \\
\hline
\end{tabular}

if and only if we can partition $S$ into subsets $S_{1}, S_{2}$ such that $\left|S_{1}\right|=\left|S_{2}\right|, \sum_{x \in S_{1}} x=\sum_{y \in S_{2}} y$, and for all $x \in S_{1}$, $b_{\alpha+x}=d$ and for all $y \in S_{2}, b_{\alpha+y}=\bar{d}(d \in\{0,1\})$.

Proof: From Theorem 1 we know that the $k$-burst error is not detected by CBT if and only if $\sum_{i=1}^{k}(-1)^{b_{\alpha+i}}(\alpha+i)=0$, i.e., $\alpha \sum_{i=1}^{k} i(-1)^{b_{\alpha+i}}+\sum_{i=1}^{k}(-1)^{b_{\alpha+i}} i=0$. But since the burst error has a zero balance signature, it follows that $\sum_{i=1}^{k}(-1)^{b_{\alpha+i}}=0$. Therefore, $\alpha \sum_{i=1}^{k}(-1)^{b_{\alpha+i}}=0$. Let $(-1)^{b_{\alpha+i}}=a_{i}$, where $a_{i} \in\{-1,1\}$. The error is not detected by CBT if and only if $\sum_{i=1}^{k} i a_{i}=0$, with half the $a_{i}$ s being 1 and the other half being -1 . The theorem now follows.

For example, suppose four bits are in error in bit positions $1,2,3$ and 4 . Then $k=4$ and $\alpha=0$. Bits $b_{1}=b_{4}=1$, and $b_{2}=b_{3}=0$. The set $S=\{1,2,3,4\}$ and we have the partition $S_{1}=\{1,4\}, S_{2}=\{2,3\}$ with $\left|S_{1}\right|=\left|S_{2}\right|$ and $1+4=2+3$. Moreover, $b_{1}=b_{4}=1$ and $b_{2}=b_{3}=0$. Therefore, the $(4,4)$-burst error is not detectable by CBT.

If $k$ is odd, then $\left|S_{1}\right| \neq\left|S_{2}\right|$, and thus all $k$ burst-errors are detected by CBT. We next consider detectability of the error when $k / 2$ is odd.

Corollary 3: If $k / 2$ is odd, then all $k$-burst errors are detected by CBT.

Proof: Consider the set $S=\{1,2, \cdots, k\}$. We know that $\sum_{x \in S} x=1+2+\cdots+k=k(k+1) / 2$. If $k / 2$ is odd then $k(k+1) / 2$ is also odd, so a bipartition of $S$ as required by Theorem 7 is not possible. Therefore, the $k$-burst error is detected by CBT.

Corollary 4: If the $k$-burst error is not detected by CBT, then $k$ must be a multiple of 4 .

For an error-free sequence of length $m$, there are $(m-k+1)$ possible $k$-burst errors. Therefore, as $k$ ranges from 1 to $m$, the number of all possible $k$-burst errors is $\sum_{k=1}^{m}(m-k+1)=$ $m(m+1) / 2$. In Table VII, we list the number of $k$-burst errors that are not detected for different values of $m$. To calculate the average values, we assume that all error-free sequences as well as all $k$-burst errors are equally likely.

We next turn to repeated-use errors. Recall that a $k \mathrm{~b}$ repeated-use error is one with the $k$ erroneous bits in positions $i+p n_{1}, i+p n_{2}, \cdots, i+p n_{k}$, where $p$ is a power of 2 . Let $E(i, p, k)$ represent a repeated-use error with $p=2^{r}$. For example, if $k=4$ and the errors are in bit positions 2,10 , 34,42 , then this is $E(2,8,4)$ with $n_{1}=1, n_{2}=1, n_{3}=4$, $n_{4}=5$. 
TABLE VII

Experimental ERror Coverage of $k$-Burst Errors $(1 \leq k \leq m)$

\begin{tabular}{||l|l|l|l|l|l||}
\hline $\begin{array}{l}\text { Number } \\
\text { of tests } \\
m\end{array}$ & $\begin{array}{l}\text { Number } \\
\text { of } k \text {-burst } \\
\text { errors }\end{array}$ & $\begin{array}{l}\text { Average number } \\
\text { of errors } \\
\text { undetected (CBT) }\end{array}$ & $\begin{array}{l}\text { Average CBT } \\
\text { evror coverage } \\
\text { (percent) }\end{array}$ & $\begin{array}{l}\text { Average number } \\
\text { of errors } \\
\text { undetected by ACT }\end{array}$ & $\begin{array}{l}\text { Average ACT } \\
\text { error coverage } \\
\text { (percent) }\end{array}$ \\
\hline 8 & 28 & 0.407 & 98.54 & 0.398 & 98.58 \\
9 & 36 & 0.667 & 98.15 & 0.476 & 98.68 \\
10 & 45 & 0.769 & 98.29 & 0.554 & 98.76 \\
11 & 55 & 0.867 & 98.42 & 0.633 & 98.85 \\
12 & 66 & 0.978 & 98.52 & 0.721 & 98.90 \\
13 & 78 & 1.120 & 98.56 & 0.816 & 98.95 \\
14 & 91 & 1.220 & 98.66 & 0.891 & 99.02 \\
15 & 105 & 1.328 & 98.74 & 0.976 & 99.07 \\
16 & 120 & 1.448 & 98.79 & 1.067 & 99.11 \\
20 & 190 & 2.014 & 98.94 & 1.496 & 99.21 \\
\hline
\end{tabular}

Theorem 8: Consider the repeated-use error $E(i, p, k)$ with zero balance signature and the errors in bits $b_{i+2^{r} n_{1}}$, $b_{i+2^{r} n_{2}}, \cdots, b_{i+2^{r} n_{k}}$. Let $S$ be the set $\left\{n_{1}, n_{2}, \cdots, n_{k}\right\}$. Then $E(i, p, k)$ is not detected by CBT if and only if we can partition $S$ into subsets $S_{1}, S_{2}$ such that $\left|S_{1}\right|=\left|S_{2}\right|$, $\sum_{x \in S_{1}} x=\sum_{y \in S_{2}} y$, and for all $x \in S_{1}, b_{i+2^{r} x}=d$ and for all $y \in S_{2}, b_{i+2^{r} y}=\bar{d}(d \in\{0,1\})$.

Corollary 5: Consider the repeated-use error $E(i, p, k)$ with the bits in error being $b_{i+2^{r} n_{1}}, b_{i+2^{r} n_{2}}, \cdots, b_{i+2^{r} n_{k}}$, and let $\sum_{i=1}^{k} n_{i}$ be odd. Then $E(i, p, k)$ is detected by CBT.

Note that if $k$ is odd, then all repeated-use errors $E(i, p, k)$ are detected by CBT. We next consider repeated-use errors that are equally spaced. We describe such errors using the parameter $t$, where $n_{j}=j t$ for an error in bit position $i+p n_{j}$. This means that the errors are in bit positions $i+t p, i+2 t p, \cdots, i+k t p$; so consecutive errors are separated by distance $t p$.

Corollary 6: Suppose the errors in the repeated-use error $E(i, p, k)$ are equally spaced, i.e. $n_{j}=j t$ for an error at the bit position $i+2^{r} n_{j}$. Then, if $k / 2$ and $t$ are odd, the error $E(i, p, k)$ is detected by CBT.

Proof: Consider the set $S=\left\{n_{1}, n_{2}, \cdots, n_{k}\right\}=$ $\{t, 2 t, \cdots, k t\}$. Now, $\sum_{x \in S} x=t+2 t+\cdots+k t=$ $t k(k+1) / 2$. If $k / 2$ and $t$ are odd, then the bipartition of $S$ required by Theorem 8 is not possible and the result follows.

\section{FAUlt COVERAGE}

The exceptionally low aliasing demonstrated in Section IV suggests that very good post-compaction fault coverage can be expected from either CBT or ACT. To investigate this issue further, we determined via simulation the coverage of irredundant SSL faults by balance testing, CBT, and ACT for the ISCAS 85 benchmark circuits.

We first performed the fault coverage experiments with both reduced and pseudorandom test patterns for the ISCAS benchmarks. The reduced test sets used were generated by the COMPACTEST ATPG program [15]. Since the number of test patterns in each set is small-COMPACTEST aims to produce small but complete test sets-the process was repeated for every primary output using a fault-dictionary-based simulation program that explicitly computes fault signatures. A fault is detected if its signature differs from the fault-free signature for at least one primary output. From Table VIII, we see that the fault coverage of CBT is $100 \%$ in all but four cases, where the coverage is slightly less. CBT performs better than balance
TABLE VIII

Fault Coverage of the ISCAS 85 Circutrs for Reduced Test Sets

\begin{tabular}{|c|c|c|c|c|c|c|c|c|}
\hline \multirow{2}{*}{$\begin{array}{l}\text { ISCAS } \\
\text { benchmark } \\
\text { circuit }\end{array}$} & \multirow{2}{*}{$\begin{array}{l}\text { Number of } \\
\text { of test } \\
\text { patterns }\end{array}$} & \multirow{2}{*}{$\begin{array}{l}\text { Number of } \\
\text { detectable } \\
\text { faults }\end{array}$} & \multicolumn{3}{|c|}{$\begin{array}{l}\text { Number of faults detected } \\
\text { after compaction }\end{array}$} & \multicolumn{3}{|c|}{$\begin{array}{l}\text { Fault coverage } \\
\text { after compaction }\end{array}$} \\
\hline & & & $\begin{array}{l}\text { Balance } \\
\text { testing }\end{array}$ & $\mathrm{CBT}$ & ACT & $\begin{array}{l}\text { Balance } \\
\text { testing }\end{array}$ & CBT & $\mathrm{ACT}$ \\
\hline$c 432$ & 48 & 520 & 485 & 520 & 520 & 93.27 & 100 & \begin{tabular}{|l|}
100 \\
\end{tabular} \\
\hline & 59 & & 70 & 75 & 750 & 94.40 & 100 & 100 \\
\hline & 30 & & & & 942 & 99.36 & & 100 \\
\hline cl355 & 95 & 15 & 1527 & 1563 & 1565 & 97.51 & 99.81 & 99.93 \\
\hline c19 & 129 & 18 & & 1865 & 1865 & 98.13 & 99.73 & 99.73 \\
\hline c2670 & 75 & & & 2626 & 2630 & 96.12 & \begin{tabular}{|l|l|}
99.84 & \\
\end{tabular} & 100 \\
\hline c35 & 113 & & & & 32 & 96.08 & & 100 \\
\hline c5315 & 59 & 52 & 49 & 31 & 52 & 92.06 & 98.29 & 100 \\
\hline$c 62$ & 23 & & & 7710 & 77 & 99.96 & 100 & 100 \\
\hline c7552 & 88 & 7419 & 7160 & 7416 & 7416 & 96.51 & 100 & 100 \\
\hline
\end{tabular}

TABLE IX

FAULT COVERAGE FOR SOME ISCAS 85 Circuits with Pseudorandom Test Patterns

\begin{tabular}{|c|c|c|c|c|c|c|c|c|c|}
\hline \multirow{2}{*}{$\begin{array}{l}\text { ISCAS } \\
\text { benchmark } \\
\text { circuit }\end{array}$} & \multirow{2}{*}{$\begin{array}{l}\text { Number of } \\
\text { of test } \\
\text { patterns }\end{array}$} & \multirow{2}{*}{$\begin{array}{l}\text { Number of } \\
\text { detectable } \\
\text { faults }\end{array}$} & \multirow{2}{*}{$\begin{array}{l}\text { Number of } \\
\text { faults detected } \\
\text { by randorn } \\
\text { patterns }\end{array}$} & \multicolumn{3}{|c|}{\begin{tabular}{|c|} 
Number of faults detected \\
after compaction
\end{tabular}} & \multicolumn{3}{|c|}{$\begin{array}{l}\text { (Detectable) coverage } \\
\text { after compaction }\end{array}$} \\
\hline & & & & $\begin{array}{l}\text { Balance } \\
\text { testing }\end{array}$ & CBT & ACT & $\begin{array}{l}\text { Balance } \\
\text { testing }\end{array}$ & CBT & ACT \\
\hline & & 520 & 520 & 515 & 520 & 520 & 99.04 & 100 & 100 \\
\hline & 1024 & 520 & 520 & 515 & 520 & $\begin{array}{l}520 \\
520\end{array}$ & 99.04 & 1000 & 100 \\
\hline C499 & & 520 & 52 & & 520 & $\begin{array}{l}520 \\
750\end{array}$ & $\begin{array}{l}99.42 \\
97.32\end{array}$ & $\begin{array}{l}100 \\
100\end{array}$ & 1000 \\
\hline & .102 & 750 & $\begin{array}{l}100 \\
750\end{array}$ & $\begin{array}{l}300 \\
730\end{array}$ & $\begin{array}{l}1100 \\
750\end{array}$ & 750 & 97.33 & 100 & 100 \\
\hline & 2048 & 750 & 750 & 72 & 750 & 750 & 96.93 & 100 & 100 \\
\hline с880 & 512 & 942 & 907 & 846 & 907 & 90 & 93.27 & 100 & 100 \\
\hline & & & 929 & 900 & 929 & 92 & 96.87 & 00 & 100 \\
\hline & 2048 & & & 855 & & & 91.63 & 0 & 100 \\
\hline e1355 & 512 & 1574 & $\mid 1163$ & 852 & 1163 & 1163 & 73.25 & 100 & \\
\hline & 1024 & 1574 & 1335 & 1106 & 1321 & 1321 & 82.85 & 98.95 & 98.95 \\
\hline c1908 & 51 & 1879 & & & 1238 & 123 & 81.27 & 100 & 100 \\
\hline
\end{tabular}

testing for all these circuits. In all but three cases, the fault coverage of CBT is equal to that of ACT.

We also obtained the CBT and ACT fault coverage with pseudorandom testing for some of the ISCAS circuits. The pseudorandom test patterns were generated using the FSIM fault simulation program [10]. For these experiments, we considered three different test sets for each of the c432, c499, and c880 benchmark circuits, and two different test sets for the $\mathrm{c} 1355$ and $\mathrm{c} 1908$ circuits. In each case, we obtained nearly $100 \%$ coverage of the detectable faults after compaction for both CBT and ACT (Table IX). The entries in the last three columns refer to the percentage of faults that are detected by random patterns as well as by the fault signature. For the larger ISCAS circuits, we were unable to perform the experiments with pseudorandom patterns because of the excessive memory required to construct the fault dictionaries. However, this does not limit the applicability of CBT because, as in other BIST methods, fault dictionaries are not required as part of the test procedure. The above experimental data strongly suggests that the drop in fault coverage for these circuits is negligible.

We also simulated CBT for some common MSI circuits [20]. Because these circuits have very few primary inputs, we used exhaustive test patterns for one set of experiments. Table X(a) shows that the fault coverage for each of these circuits is $100 \%$. The fault coverage for balance testing drops considerably when reduced test sets generated by COMPACTEST are applied. This is illustrated in Table $\mathrm{X}(\mathrm{b})$.

The experimental data presented in Tables VIII-X complement the error coverage analysis of Section IV. These results show that CBT is very useful for testing circuits for which a high degree of test confidence is required. In practice, SSL fault coverage of $99 \%$ or more is often mandated, and CBT is 
TABLE X

Fault Coverage for Some Common MSI Circuits with (a) Exhaustive AND (b) Reduced Test Sets

\begin{tabular}{|c|c|c|c|c|c|c|c|c|}
\hline \multirow{2}{*}{\begin{tabular}{|l|} 
MSI \\
circuit
\end{tabular}} & \multirow{2}{*}{$\begin{array}{l}\text { Number of } \\
\text { of test patterns } \\
\text { (exhaustive) }\end{array}$} & \multirow{2}{*}{$\begin{array}{l}\text { Number of } \\
\text { detectabie } \\
\text { faults }\end{array}$} & \multicolumn{3}{|c|}{ Number of faults detected } & \multicolumn{3}{|c|}{ Fault coverage } \\
\hline & & & $\begin{array}{l}\text { Balance } \\
\text { testing }\end{array}$ & CBT & ACT & $\begin{array}{l}\text { Balance } \\
\text { testing }\end{array}$ & CBT & ACT \\
\hline 74152 & 2048 & 106 & 100 & 106 & 10 & 94.35 & 100 & 100 \\
\hline & 20 & & & 21 & & 100 & & 10u \\
\hline & & & & 20 & 2 & 72.59 & 100 & 100 \\
\hline 74181 & 16384 & 384 & 380 & 384 & 384 & 98.96 & 100 & 100 \\
\hline
\end{tabular}

(a)

\begin{tabular}{|c|c|c|c|c|c|c|c|c|}
\hline \multirow{2}{*}{\begin{tabular}{|l|} 
MSI \\
circuit
\end{tabular}} & \multirow{2}{*}{$\begin{array}{l}\text { Number of } \\
\text { of test patterns } \\
\text { (reduced) }\end{array}$} & \multirow{2}{*}{$\begin{array}{l}\text { Number of } \\
\text { detectable } \\
\text { faults }\end{array}$} & \multicolumn{3}{|c|}{ Number of faults detected } & \multicolumn{3}{|c|}{ Fault coverage } \\
\hline & & & $\begin{array}{l}\text { Balance } \\
\text { testing }\end{array}$ & CBT & ACT & $\begin{array}{l}\text { Balance } \\
\text { testing }\end{array}$ & CBT & ACT \\
\hline 74152 & 16 & 106 & 106 & \begin{tabular}{|l|}
106 \\
\end{tabular} & 106 & 100 & 100 & 100 \\
\hline $74 \mathrm{~L} 85$ & 25 & 21 & 10 & 21 & 2 & 47.69 & 100 & 100 \\
\hline 74283 & 13 & 208 & 109 & 208 & 208 & 52.40 & 100 & 100 \\
\hline 74181 & 18 & 384 & 95 & 384 & 384 & 24.75 & 100 & 100 \\
\hline 74181 & 12 (minimal) & 384 & 94 & 384 & 384 & 24.48 & 100 & 100 \\
\hline
\end{tabular}

(b)

able to achieve that without requiring either exhaustive testing or a long signature.

\section{CONCLUSIONS}

Cumulative balance testing appears to a useful method for signature-based BIST that generalizes both balance testing and ACT. CBT provides greater fault coverage than balance testing, and in most cases, the same fault coverage as ACT, but with a shorter fault signature. The SSL fault coverage of CBT for most of the ISCAS 85 benchmark circuits and some common MSI circuits in the $74 \mathrm{X}$ series is the same as that for ACT. For all but four of the ISCAS circuits, the fault coverage is $100 \%$, and for all but one circuit, the fault coverage is over $99.5 \%$. We analyzed the error coverage for CBT under the independent errors assumption using the central limit theorem. Our analysis, which also applies to ACT, shows an exceptionally high error coverage-over $99 \%$ even for short test lengths. The hardware overhead of CBT consists of the response compression logic, and routing and multiplexing costs. For processor circuits, the former can be reduced by utilizing existing datapath modules like adders and counters. The problem of routing can be addressed by using efficient place-and-route CAD tools. The provably high error and fault coverage for the benchmark circuits appear to make CBT suitable for testing circuits when a fault coverage of $99 \%$ or more is required.

\section{ACKNOWLEDGMENT}

The authors are grateful to Profs. I. Pomeranz and S. Reddy of the University of Iowa for providing us with COMPACTEST. Thanks also go to Prof. D. Ha of Virginia Tech. for providing us with FSIM.

\section{APPENDIX}

We first derive an upper bound on $\delta C_{m}$, the difference between the fault-free and faulty cumulative balance signatures.

Lemma 2: Let $k$ b of an $m$ b response sequence be in error in positions $i_{1}, i_{2}, \cdots, i_{k}$. Let the fault-free value of the bit in position $i_{j}$ be $b_{i_{j}}$, and let the difference between the fault-free and faulty cumulative balance signatures be $\delta C_{m}$. Then

$$
\delta C_{m} \leq \begin{cases}\left\lfloor m^{2} / 4\right\rfloor, & \text { if } \sum_{j=1}^{k}(-1)^{b_{i_{j}}} \geq 0 \\ \left\lfloor m^{2} / 4+m / 2\right\rfloor, & \text { if } \sum_{j=1}^{k}(-1)^{b_{i_{j}}}<0 .\end{cases}
$$

Proof: We consider the following three cases:

Case 1: $\sum_{j=1}^{k}(-1)^{b_{i}}=0$. Then $\delta C_{m} \leq m+(m-1)+$ $\cdots+(m / 2+1)-(1+2+\cdots+m / 2)=m^{2} / 4$, if $m$ is even, and $\delta C_{m} \leq m+(m-1)+\cdots+(m+3) / 2+1-(1+$ $2+\cdots+(m-1) / 2)=\left(m^{2}-1\right) / 4$, if $m$ is odd. This yields $\delta C_{m} \leq\left\lfloor m^{2} / 4\right\rfloor$.

Case 2: $\sum_{j=1}^{k}(-1)^{b_{i_{j}}}=u>0 . \delta C_{m}$ is maximum when the first $x$ (whose value is to be determined) bits of the response sequence are 1 and in error, and the last $x+u$ b of the response sequence are 0 and in error. This yields

$$
\begin{aligned}
\delta C_{m} \leq & f(x) \\
= & \frac{m(m+1)}{2}-\frac{(m-x-u)(m-x-u+1)}{2} \\
& -\frac{x(x+1)}{2}-(m+1) u .
\end{aligned}
$$

Differentiating $f(x)$ with respect to $x$, we get $\frac{d f(x)}{d x}=$ $m-u-2 x$, which is 0 if $x=(m-u) / 2$. Since $\frac{d^{2} f(x)}{d x^{2}} \leq 0$, $f(x)$ is maximum for $x=(m-u) / 2$. Substituting this value of $x$ in (8), we obtain $\delta C_{m} \leq m^{2} / 4-\left(m u+u^{2}+2 u\right) / 4 \leq m^{2} / 4$.

Case 3: $\sum_{j=1}^{k}(-1)^{b_{i_{j}}}=u<0 . \delta C_{m}$ is maximum when the last $x$ (value to be determined) $\mathrm{b}$ of the response sequence are 0 and in error, and the last $x+u \mathrm{~b}$ of the response sequence are 1 and in error. This gives

$$
\begin{aligned}
\delta C_{m} \leq f(x)= & \frac{m(m+1)}{2}-\frac{(m-x)(m-x+1)}{2} \\
& -\frac{(x+u)(x+u+1)}{2}+(m+1) u .
\end{aligned}
$$

Differentiating $f(x)$ with respect to $x$, we get $\frac{d f(x)}{d x}=$ $m-u-2 x$ which is 0 if $x=(m-u) / 2$. Since $\frac{d^{2} f(x)}{d x^{2}}<0$, $f(x)$ is maximum for $x=(m-u) / 2$. Substituting this value of $x$ in (9), we obtain $\delta C_{m} \leq m^{2} / 4+m / 2$.

We next state the central limit theorem, which is used in Section IV to study the distribution of $X=X_{1}+X_{2}+\cdots+$ $X_{m}$.

Theorem 9: (Central Limit Theorem [9]) If $X_{1}, X_{2}, \cdots$, $X_{m}$ are independent random variables, where $X_{i}(1 \leq i \leq m)$ has mean $\mu_{i}$ and variance $\sigma_{i}^{2}$, then under certain regularity conditions, the limiting distribution of $X=\sum_{i=1}^{m} X_{i}$ is normal with mean $\sum_{i=1}^{m} \mu_{i}$ and variance $\sum_{i=1}^{m} \sigma_{i}^{2}$.

The random variables $X_{1}, X_{2}, \cdots, X_{m}$ are independent. To study the limiting distribution of $X$ using the central limit theorem, we have to prove that $X_{1}, X_{2}, \cdots, X_{m}$ satisfy the Lindberg condition [9].

Theorem 10: (Lindeberg Theorem [9]) For the set of independent random variables $X_{1}, X_{2}, \cdots, X_{m}$, where $X_{i}(1 \leq$ $i \leq m$ ) has mean $\mu_{i}$ and variance $\sigma_{i}^{2}$, the central limit theorem holds whenever for every $\epsilon>0$ the truncated variables $U_{k}$ 


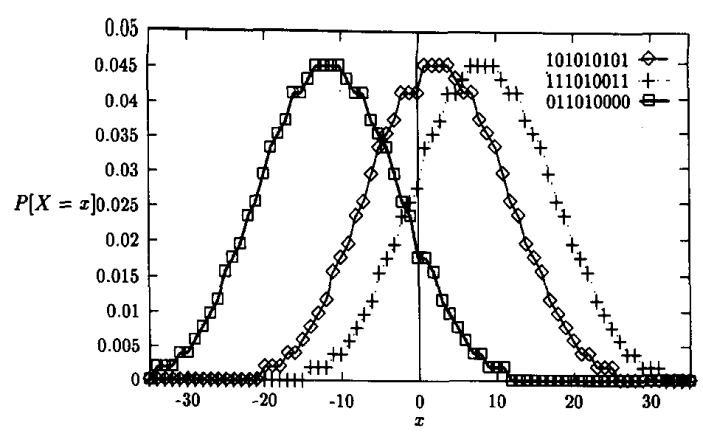

(a)

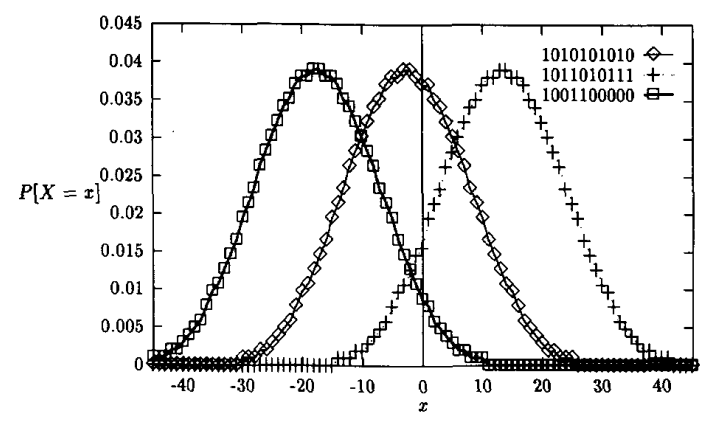

(b)

Fig. 8. The probabilities $P[X=x]$ obtained experimentally for three arbitrarily chosen fault-free sequences with (a) $m=9$ and (b) $m=10$.

$(1 \leq k \leq m)$ defined by

$$
U_{k}= \begin{cases}X_{k}-\mu_{k}, & \text { if }\left|X_{k}-\mu_{k}\right| \leq \epsilon s_{m} \\ 0, & \text { otherwise }\end{cases}
$$

satisfy the conditions $\lim _{m \rightarrow \infty} s_{m}^{2}=\infty$, and

$$
\lim _{m \rightarrow \infty} \frac{1}{s_{m}^{2}} \sum_{k=1}^{m} E\left[U_{k}^{2}\right]=1
$$

where $s_{m}^{2}=\sum_{i=1}^{m} \sigma_{i}^{2}$.

The first part of the Lindeberg condition follows directly for both the independent and asymmetric error models from the expressions for $\sigma_{i}^{2}$ derived in Section IV. Here, we prove the second part. For the independent error model, $s_{m}^{2} \approx \frac{2 m^{3} \alpha^{2}}{3}$ and $\mu_{k}=0$. If $\epsilon=\infty, U_{k}=X_{k}$ for every value of $m$, hence $\frac{1}{s_{m}^{2}} \sum_{k=1}^{m} E\left[X_{k}^{2}\right]=1$, and the Lindberg condition is satisfied. Next suppose $0<\epsilon<\infty$. We know that $\left|X_{k}\right| \leq m$, and therefore we pose the following question: Given $0<\epsilon<\infty$, for what value(s) of $m$ is $\epsilon s_{m}>m$ ? Now, $\epsilon s_{m}>m$ implies $\epsilon \sqrt{\frac{2 m^{3} \alpha^{2}}{3}}>m$, which gives us $m>\frac{3}{2 \alpha^{2} \epsilon^{2}}$. Let $m_{\epsilon}=\left\lceil\frac{3}{2 \alpha^{2} \epsilon^{2}}\right\rceil$. Then, for $m>m_{\epsilon}$, the $U_{k}$ 's are identical with the $X_{k}$ 's. This implies that there exists $m_{\epsilon}<\infty$ such that for all $m>m_{\epsilon}, \frac{1}{s_{m}^{2}} \sum_{k=1}^{m} E\left[U_{k}^{2}\right]=\frac{1}{s_{m}^{2}} \sum_{k=1}^{m} E\left[X_{k}^{2}\right]=$ $\frac{1}{s_{m}^{2}} \cdot s_{m}^{2}=1$; therefore, $\lim _{m \rightarrow \infty} \frac{1}{s_{m}^{2}} \sum_{k=1}^{m} E\left[U_{k}^{2}\right]=1$. Honco, the second part of the Lindberg condition is satisified. For the asymmetric error model, $s_{m}^{2} \approx \frac{m^{3}}{3}\left(\theta_{1}+\theta_{0}+2 \theta_{1} \theta_{0}-\theta_{1}^{2}-\theta_{0}^{2}\right)$, $\mu_{k}=k\left(\theta_{1}-\theta_{0}\right)$, and $\left|X_{k}-\mu_{k}\right| \leq m$, so that for $m>$ $3 /\left(m^{2}\left(\theta_{1}+\theta_{0}+2 \theta_{1} \theta_{0}-\theta_{1}^{2}-\theta_{0}^{2}\right) \epsilon^{2}\right)$, the truncated $U_{k}$ variables are once again identical with the $X_{k}$ 's. Therefore, the Lindeberg condition is satisfied for the asymmetric error model.

In Fig. 7, we see that the distribution of the random variable $X$ fits the normal curve for values of $m$ as low as 11. Fig. 8 shows that the approximation is slightly less accurate for $m=9$ and $m=10$. However, it improves rapidly as $m$ increases. Note that for the ISCAS benchmarks (Table V), $m \geq 23$ in all cases, and $m$ has an average value of 72 .

\section{REFERENCES}

[1] S. B. Akers, "A parity bit signature for exhaustive testing," IEEE Trans. Comput.-Aided Design, vol. 7, pp. 333-338, Mar. 1988.

[2] M. Abramovici, M. A. Breuer, and A. D. Friedman. Digital Systems Testing and Testable Design. New York: Computer Science Press, 1990.

[3] B. Bose, "Group-theoretic signature analysis," IEEE Trans. Comput., vol. 39, pp. 1398-1403, Nov. 1990.

[4] F. Brglez and H. Fujiwara, "A neutral netlist of 10 combinational benchmark circuits and a target simulator in Fortran," in Proc. IEEE Int. Symp. Circuits, Syst., 1985, pp. 695-698.

[5] K. Chakrabarty and J. P. Hayes, "Balance testing of logic circuits," in Proc. IEEE Int. Symp. Fault-Tolerant Computing, 1993, pp. 350-359.

[6] K. Chakrabarty and J. P. Hayes, "DFBT: A design for testability method based on balance testing," in Proc. Design Automat. Conf., 1994, pp. $351-357$.

[7] _ "Efficient test response compression for multiple-output circuits,' in Proc. IEEE Int. Test Conf., 1994, pp. 501-510.

[8] R. David, "Comments on 'Signature analysis for multiple output circuits'," IEEE Trans. Comput., vol. 39, pp. 287-288, Feb. 1990.

[9] W. Feller, An Introduction to Probability Theory and its Applications, 3rd ed., vol. 1. New York: Wiley, 1967.

[10] H. K. Lee and D. S. Ha, "An efficient forward fault simulation algorithm based on the parallel pattern single fault propagation," in Proc. IEEE Int. Test Conf., 1991, pp. 946-955.

[11] E. J. McCluskey, "Verification testing-A pseudoexhaustive test technique,"' IEEE Trans. Comput., vol. 33, pp. 541-546, June 1984.

[12] E. J. McCluskey and S. Bozorgui-Nesbat, "Design for autonomous test,"' IEEE Trans. Comput., vol. 30, pp. 866-875, Nov. 1981.

[13] D. K. Pradhan and S. K. Gupta, "A new framework for designing and analyzing BIST techniques and zero aliasing compression," IEEE Trans. Comput., vol. 40, pp. 743-763, June 1991.

[14] S. Pilarski and K. J. Wiebe, "Counter-based compaction: An analysis for BIST," J. Electron. Testing: Theory, Applicat., vol. 3, pp. 33-43, 1992.

[15] I. Pomeranz, L. N. Reddy, and S. M. Reddy, "COMPACTEST: A method to generate compact test sets for combinational circuits," in Proc. IEEE Int. Test Conf., 1991, pp. 194-203.

[16] J. E. Smith, "Measures of effectiveness of fault signature analysis," IEEE Trans. Comput., vol. 29, pp. 510-514, June 1980.

[17] R. Srinivasan, S. K. Gupta, and M. A. Breuer, "An efficient partitioning strategy for pseudo-exhaustive testing," in Proc. Design Automat. Conf., 1993, pp. 242-248.

[18] J. Rajski and J. Tyszer, "Test response compression in accumulators with rotate-carry adders," IEEE Trans. Comput.-Aided Design, vol. 12, pp. 531-539, Apr. 1993.

[19] N. R. Saxena and J. P. Robinson, "Accumulator compression testing", IEEE Trans. Comput., vol. 35, pp. 317-321, Apr. 1986

[20] Texas Instruments, The TTL Data Book, Dallas, TX, 1988.

[21] T. W. Williams, W. Daehn, M. Gruetzner, and C. W. Starke, "Aliasing errors in signature analysis registers" IEEE Design, Test Comput., vol. 4, pp. 39-45, Apr. 1987.

[22] D. Xavier, R. C. Aitken, A. Ivanov, and V. K. Agarwal, "Using an asymmetric error model to study aliasing in signature analysis registers," IEEE Trans. Comput.-Aided Design, vol. 11, pp. 16-25, Jan. 1992. 
[23] Y. You and J. P. Hayes, "Implementation of VLSI self-testing by regularization," IEEE Trans. Comput.-Aided Design, vol. 7, pp. 1261-1271, Dec. 1988.

[24] Y. Zorian and A. Ivanov, "Programmable space compaction for BIST," in Proc. IEEE Int. Symp. Fault-Tolerant Computing, 1993, pp. 340-349.

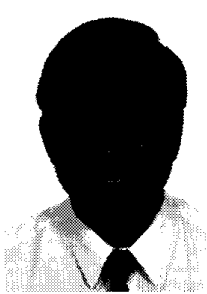

Krishnendu Chakrabarty (S'92) received the B.Tech. degree in 1990 from the Indian Institute of Technology, Kharagpur, and the M.S.E. degree in 1992 from the University of Michigan, Ann Arbor, both in computer science and engineering. He is working toward the Ph.D. degree in the Department of Electrical Engineering and Computer Science at the University of Michigan.

From 1990 to the present, he has been a Research Assistant at the University of Michigan, working in the areas of built-in self testing and design for testability. His other research interests include VLSI design, test generation, logic synthesis and verification, and fault-tolerant computing.

Mr. Chakrabarty is a student member of ACM and an associate member of Sigma Xi.

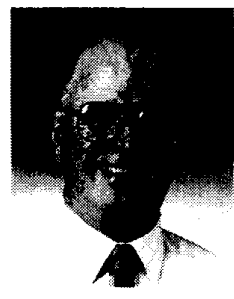

John P. Hayes (S'67-M'70-SM'81-F'85) received the B.E. degree from the National University of Ireland, Dublin, in 1965, and the M.S. and Ph.D. degrees from the University of Illinois, UrbanaChampaign, in 1967 and 1970, respectively, all in electrical engineering.

While at the University of Illinois, he participated in the design of the ILLIAC III computer. In 1970 he joined the Operations Research Group at the Shell Benelux Computing Center in The Hague where he worked on mathematical programming. From 1972 to 1982 , he was a faculty member of the Departments of Electrical Engineering-Systems and Computer Science of the University of Southern California, Los Angeles. Since 1982 he has been a Professor in the Electrical Engineering and Computer Science Department of the University of Michigan, Ann Arbor. He was the founding director of the Advanced Computer Architecture Laboratory at the University of Michigan. He is the author of over 125 technical papers and five books, including Computer Architecture and Organization, (2nd ed., New York: McGraw-Hill, 1988) Layout Minimization for CMOS Cells, (Kluwer, 1992; coauthored with R. L. Maziasz), and Introduction to Digital Logic Design, (Addison-Wesley, 1993). He has served as editor of several technical journals, including the Communications of the ACM, the IEEE TRANSACTIONS ON PARALlel AND DISTRIBUTED SYSTEMS, and the Journal of Electronic Testing. His research interests are in the areas of computer architecture, computer-aided design and testing, VLSI design, parallel processing, and fault tolerance.

Dr. Hayes was Technical Program Chairman of the 1977 International Conference on Fault-Tolerant Computing (Los Angeles, CA) and the 1991 International Computer Architecture Symposium (Toronto, Ontario, Canada). $\mathrm{He}$ is a member of the Association for Computing Machinery and Sigma Xi. 